\title{
BMJ Open Effect of national HIV testing recommendations and local interventions on HIV testing practices in a Swiss university hospital: a retrospective analysis between 2012 and 2015
}

\author{
Tosca Lazzarino, ${ }^{1}$ Sebastien Martenet, ${ }^{2}$ Rachel Mamin, ${ }^{3}$ Renaud A Du Pasquier, ${ }^{4}$ \\ Solange Peters, ${ }^{5}$ Matthieu Perreau, ${ }^{3}$ Olivier Muller, ${ }^{6}$ Olivier Hugli, ${ }^{7}$
} Matthias Cavassini, ${ }^{8}$ Katharine Elizabeth Anna Darling ${ }^{8}$

To cite: Lazzarino T, Martenet S, Mamin R, et al. Effect of national HIV testing recommendations and local interventions on HIV testing practices in a Swiss university hospital: a retrospective analysis between 2012 and 2015. BMJ Open 2018;8:e021203. doi:10.1136/ bmjopen-2017-021203

- Prepublication history and additional material for this paper are available online. To view these files, please visit the journal online (http://dx.doi.org/10.1136 bmjopen-2017-021203).

Part of this work was presented as a poster at the 27thECCMID meeting in Vienna, Austria, 2225 April 2017.

Received 20 December 2017 Revised 15 April 2018 Accepted 7 June 2018

Check for updates

(C) Author(s) (or their employer(s)) 2018. Re-use permitted under CC BY-NC. No commercial re-use. See rights and permissions. Published by BMJ.

For numbered affiliations see end of article.

Correspondence to Dr Katharine Elizabeth Anna Darling;

Katharine.Darling@chuv.ch

\section{ABSTRACT}

Objectives Despite HIV testing recommendations published by the Federal Office of Public Health (FOPH) since 2007, many individuals living with HIV are diagnosed late in Switzerland. The aim of this study is to examine the effect of the 2013 FOPH HIV testing recommendations on HIV testing rates.

Setting Ten clinical services at Lausanne University Hospital, Lausanne, Switzerland.

Participants Patients attending between 1 January 2012 and 31 December 2015.

Design Retrospective analysis using two existing hospital databases. HIV testing rates calculated as the percentage of tests performed (from the Immunology Service database) per number of patients seen (from the central hospital database).

Primary and secondary outcome measures The primary outcome was testing rate change following the 2013 FOPH testing recommendations, comparing testing rates 2 years before and 2 years after their publication. Secondary outcomes were demographic factors of patients tested or not tested for HIV.

Results 147884 patients were seen during the study period of whom $9653(6.5 \%)$ were tested for HIV, with 34 new HIV diagnoses. Mean testing rate increased from $5.6 \%$ to $7.8 \%$ after the recommendations ( $p=0.001$ ). Testing rate increases were most marked in services involved in clinical trials on HIV testing, whose staff had attended training seminars on testing indications and practice. Testing rates were lower among older (aged $>50$ years), female and Swiss patients compared with younger, male and non-Swiss patients, both globally $(p=0.001)$ and in specific clinical services.

Conclusions This simple two-database tool demonstrates clinical services in which HIV testing practice can be optimised. Improved testing rates in services involved in clinical trials on testing suggest that local engagement complements the effect of national recommendations. While, overall, HIV testing rates increased significantly over time, testing rates were lower among patients with
Strengths and limitations of this study

- A simple two-database tool was used to calculate HIV testing rates over a wide range of clinical services.

- The single-centre design enabled knowledge of clinical service structure and therefore the profile of patients being examined.

- Testing rates could be compared within clinical services over time.

- Although the two-database tool is simple and robust, it does not provide a 'margin of improvement' for testing rates, as it examines only testing rates and not the parameters which could influence these.

- Due to the retrospective nature of this study, the denominator taken to calculate testing rates was the number of patients visiting each service, rather than the number of patients visiting the service who presented testing indications. Changes in testing rates within a service could therefore be due to changes in the number of patients meeting screening criteria rather than changes in testing practice per se.

similar demographic profiles to individuals diagnosed late in Switzerland.

\section{INTRODUCTION}

HIV testing is key in diagnosing individuals living with HIV and in enabling engagement in care, antiretroviral treatment and viral suppression. Testing individuals early in infection reduces consequent morbidity, mortality, healthcare costs and onward transmission. ${ }^{1}$ HIV testing recommendations have been published in Switzerland by the Federal Office of Public Health (FOPH) since 2007, ${ }^{2}$ with updates in 2010, 2013 and $2015 .^{3-5}$ The 
recommendations propose physician-initiated counselling and testing (PICT) which is targeted and opt-in, and requires testing to be offered to patients explicitly before being performed. The FOPH recommendations present the situations in which HIV testing is indicated, notably, symptoms and signs of acute HIV infection, AIDS-defining illness, HIV indicator diseases and groups at high risk of HIV acquisition. The physician is responsible for recognising these situations in clinical practice.

In contrast to PICT, the HIV testing recommendations of the USA, ${ }^{6}$ France $^{7}$ and the $\mathrm{UK}^{8}$ propose non-targeted and opt-out testing in healthcare settings in which local HIV seroprevalence is above a certain threshold $(0.1 \%$ in the USA and France, $0.2 \%$ in the UK). In this case, testing is performed regardless of clinical presentation or risk factors, unless the patient explicitly declines. Patient-related, doctor-related and system-related barriers exist to both targeted and non-targeted testing approaches ${ }^{9-11}$ and may delay HIV diagnosis in positive individuals. In Switzerland, almost half of the patients newly diagnosed with HIV are identified late, with CD4 counts below 350 cells $/ \mathrm{mm}^{3}$ or an AIDS-defining condition, ${ }^{12}$ suggesting that testing practice is suboptimal.

In 2012, our group examined the effect of the 2010 FOPH HIV testing recommendations on the testing rates of selected clinical services at Lausanne University Hospital (LUH), Lausanne, and reported that there was no significant change in HIV testing practice associated with the recommendations' publication. ${ }^{13}$ Among the clinical services, the emergency department (ED) and the oncology service had particularly low testing rates at, respectively, $1 \%$ and $4 \%$ of all patients seen. ${ }^{13}$ Following the 2010 FOPH recommendations, which specifically mentioned the ED as a service in which HIV testing should take place, we observed that $82 \%$ of ED doctors in Frenchspeaking Switzerland were unaware of national HIV testing recommendations and that even those aware did not always propose testing when indicated. ${ }^{14}$ After providing training seminars on testing indications and the practical aspects of testing to address this lack of awareness, we observed other ED doctor-related barriers to proposing testing, notably, a preference to focus on the reason(s) for presenting over discussing HIV. ${ }^{11}$ In the oncology service, we observed that HIV testing of patients diagnosed with AIDS-defining cancers (ADCs) was not universal and was especially poor among patients with invasive cervical cancer (HIV testing rate $11 \%) .{ }^{15}$ To investigate barriers to HIV testing in this service, we provided information seminars for doctors and nurses on HIV testing indications and testing practice, and examined HIV testing rates among patients newly diagnosed with non-ADCs. Among 239 patients enrolled in this study, the HIV testing rate increased from 4\% (baseline) to $18 \%$, and patient acceptance of HIV testing offered was high $(91 \%) .^{16}$

In November 2013, the FOPH HIV testing recommendations were updated, differing from the 2010 recommendations in three main ways. ${ }^{4}$ First, testing indications were graded such that HIV testing should be expressly recommended (for acute HIV infection and AIDS-defining conditions), recommended (for HIV indicator diseases, following the results of the HIV Indicator Diseases across Europe Study ${ }^{17}$ ) or proposed (where not diagnosing HIV could have severe consequences or for individuals at risk of HIV acquisition). Second, patients undergoing immunosuppressive therapy, including chemotherapy, were included as a group in whom HIV testing should be proposed. Third, it was stated explicitly that not performing an HIV test when indicated could have medicolegal consequences.

The aim of this study was to repeat the analysis of HIV testing practice in selected clinical services at our centre, this time examining the effect on testing rates of the 2013 FOPH HIV testing recommendations.

\section{METHODS}

\section{Patient and public involvement}

Patients and public were not involved in the design of this study. The research question on HIV testing practice arose from the experience of some of our patients attending our HIV OP service of presenting late with HIV despite having visited different services at our hospital during the years preceding their HIV diagnosis.

All patient data used were stripped of identifiers prior to analysis.

\section{HIV testing}

HIV testing performed in the Immunology Service uses a fourth generation screening assay to detect anti-HIV1/2 antibodies and p24 antigen (Cobas Elecsys HIV combi PT, Roche Diagnostics, Rotkreutz, Switzerland). Reactive samples undergo confirmation assays (p24 neutralisation assay and a line immunoassay for HIV-1/2 antibodies) before the release of a positive result as previously described. ${ }^{13}$ All HIV tests performed are entered into the Immunology Service database, together with the requesting service, date of request, date of test and result.

\section{Study setting}

The study was retrospective and single-centre, performed at LUH, Lausanne, Switzerland, a 1500-bed teaching hospital where local HIV seroprevalence is estimated to be between $0.2 \%$ (estimated from Swiss HIV Cohort Study data) and $0.5 \%$ (UNAIDS estimation). ${ }^{18} 19$ At this centre, doctors attend regular educational seminars as is standard in a teaching hospital but without necessarily a focus on HIV.

Ten clinical services were selected for analysis: general internal medicine inpatients (IP), neurology IP, cardiology IP, intensive care units, ED, psychiatry IP (excluding services for substance misuse where HIV testing is routine), oncology outpatients (OP), dermatology IP and OP, ear, nose and throat (ENT) surgery IP and OP, and non-ENT surgery IP (including vascular, cardiothoracic, gastrointestinal, maxillofacial surgery, urology and neurosurgery). The services were selected for $\geq 1$ of the following 
reasons: (1) receiving patients likely to present with HIV testing indications by specialty ${ }^{3}$; (2) previous target of educational interventions (ED, oncology service ${ }^{11} 141620$ ); and (3) enabling comparison with testing rates prior to $2012 .{ }^{13}$ Due to the retrospective nature of this study, it was not possible to identify patients presenting specific HIV testing indications. In order to make the number of patients presenting as close as possible to the number of patients presenting with testing indications, we restricted our analysis to patients presenting to selected subunits within each clinical service. For example, for the cardiology service, we included subunits receiving acute ischaemic heart disease admissions and excluded subunits related to congenital anomalies or cardiac transplant work-ups.

Between 2012 and 2015, the oncology service underwent expansion, resulting in some units seeing a substantial increase in patient turnover. For the current study, we restricted analysis to units examined previously $^{13}$ and those taking part in clinical trials on HIV testing.

\section{Study design}

All patients aged $\geq 16$ years old presenting to the selected clinical services between 1 January 2012 and 31 December 2015 were eligible for inclusion. Using the central hospital database, the number of patients attending each service was obtained over four 12-month blocks, corresponding to each calendar year. Dates of all hospital visits and patient demographic parameters (age, sex, origin) were also obtained from this database. The number of HIV tests performed during the same 12-month blocks was obtained from the Immunology Service database.

For each 12-month block, HIV testing rates were calculated as the percentage of tests performed per number of patients seen. For the testing rate calculations, the number of patients rather than the number of clinical episodes was taken as the denominator, to avoid underestimating testing rates in clinical services which may see the same patient several times, notably in the OP sector.

For the ED, to examine the effect of local clinical trials and associated educational interventions on HIV testing, testing rates were calculated monthly in addition to 12-monthly. For 12-monthly testing rates, the entire service was analysed whereas for the monthly testing rates, analysis was restricted to the sections involved in the clinical trials. In the oncology service, testing rates were additionally compared between 2012 and 2013, in line with a clinical trial which ran from July to October 2013. ${ }^{16}$ However, as the 2013 FOPH recommendations introduced 'proposing' testing to all patients undergoing immunosuppressive treatment, the specific effect of clinical trials after 2013 was not examined in this study. The results of these trials will be published elsewhere (manuscript in preparation).

\section{Positive HIV tests}

Occasionally, an HIV test (the fourth generation screening assay described above) is requested erroneously for patients of known positive HIV status instead of viral load. For all tests conducted and confirmed as positive, therefore, test dates were cross-referenced with electronic medical records to determine whether each positive test was a new or a known positive case.

\section{Statistical analyses}

Data are presented as means with $\mathrm{SD}$, medians with $\mathrm{IQR}$ and as percentages. Means were compared using Student's t-test and proportions compared using the $\chi^{2}$ test using two-way contingency tables. To examine the effect of the 2013 FOPH HIV testing recommendations, testing rates for each clinical service, and overall, were examined during the 2 years before and the 2 years after the publication of the recommendations (1 January 2012 to 31 December 2013 and 1 January 2014 to 31 December 2015) and then compared. Although the updated FOPH recommendations were published in November 2013, the month of December 2013 was included in the 'before' analysis given that there is usually a lag period of at least 1 month between recommendations being published and being read and/or implemented. ${ }^{13}$ Oncology service testing rates were additionally compared between 1 January to 31 December 2012 and 1 January to 31 December 2013.

In our 2012 report on testing, we commented that patient origin may have an effect on testing, based on the observation that the medical $\mathrm{OP}$ service not examined in the present study had a particularly high percentage of patients from countries with high HIV prevalence (estimated to be up to $65 \%{ }^{13}$ ). We therefore examined the effect of patient age, sex and origin on testing rates in each clinical service, and overall. Patient origin was categorised as Swiss, from neighbouring countries (France, Germany, Austria, Italy and Lichtenstein), from sub-Saharan Africa (SSA) and 'other', comprising countries not falling into the first three categories. When patient numbers were low in specific origin categories, analyses were performed by grouping patients in the latter three categories as 'non-Swiss'. Patients lacking data on origin were removed from analyses provided the proportion of patients removed was $<10 \%$; if the percentage was $\geq 10 \%$ in a specific service, analysis of origin data was not performed.

All analyses were performed using Microsoft Excel 2008 (Microsoft, Redmond, Washington, USA).

\section{RESULTS}

\section{Study population}

During the 4-year study period, 147884 patients were seen at the 10 clinical services examined. Patient demographic profile did not change significantly during this time. In total, 9653 patients underwent HIV testing (global HIV testing rate $6.5 \%)$. Online supplementary table 1 shows 
the demographic characteristics of patients tested and not tested.

The total number of patients attending the 10 clinical services increased progressively over the 4-year period from 34861 to 36460 patients but the difference in the number of patients attending before versus after the 2013 FOPH recommendations was not significant $(\mathrm{p}=0.14)$.

\section{HIV tests and testing rates}

The total number of tests performed increased over the 4-year period, with a mean of $2220 \pm 94.5$ tests before and $2803 \pm 171$ tests after the 2013 FOPH recommendations $(\mathrm{p}=0.001)$. The global testing rates (for all clinical services together) also increased significantly between before and after the 2013 recommendations from $5.6 \% \pm 0.2 \%$ (2012$2013)$ to $7.8 \% \pm 0.4 \%(2014-2015)(\mathrm{p}=0.002) \quad$ (table 1 and figure 1). Testing rate increases were particularly marked in the ED (from $4.2 \% \pm 0.3 \%$ to $5.6 \% \pm 0.4 \%, \mathrm{p}=0.001$, table 1 ) and the oncology service (from $3.8 \% \pm 0.8 \%$ to $23.6 \% \pm 7.4 \%, \mathrm{p}=0.0001$, figure 1 ).

\section{Positive HIV tests}

In total, 18 new HIV diagnoses $(0.46 \%$ of tests performed) were made between 2012 and 2013, and 16 new diagnoses ( $0.33 \%$ of tests performed) were made between 2014 and 2015 (table 1). The demographic characteristics have not differed significantly between the two time periods and so are presented here for all 34 newly diagnosed patients: median age was 42 years (IQR 34-48), 28 (82\%) were men, $14(41 \%)$ were Swiss and 20 were non-Swiss, including eight (24\%) from SSA. Median CD4 count at diagnosis was 188 cells $/ \mathrm{mm}^{3}$ (IQR 32-363).

There were 30 erroneous repeat HIV tests in patients of known positive status. However, retesting enabled two patients who had been lost to follow-up to be reintegrated into care.

The rate of positive tests out of tests performed remained stable with time in each service, at between $0 \%$ and $0.9 \%$ (table 1), with no change between before and after the 2013 FOPH recommendations $(\mathrm{p}=0.77)$. There were no positive tests among patients attending the services of cardiology, surgery and oncology, although testing rates in the cardiology and surgery services were at the lower end of all services studied (figure 1).

\section{Effect of patient demographic profile on testing}

The breakdown of patients by age, sex and origin was examined using the two study databases. Globally, the proportion of patients attending the 10 clinical services who were aged below 50 did not change over the 4-year period, with a mean proportion of $31.8 \% \pm 0.24 \%$. Equally, the proportion of patients aged below 50 who were tested for HIV remained stable with a mean proportion of $51.3 \% \pm 1.36 \%$. However, in some services, the proportion of patients tested was higher among patients aged below 50 than among older patients

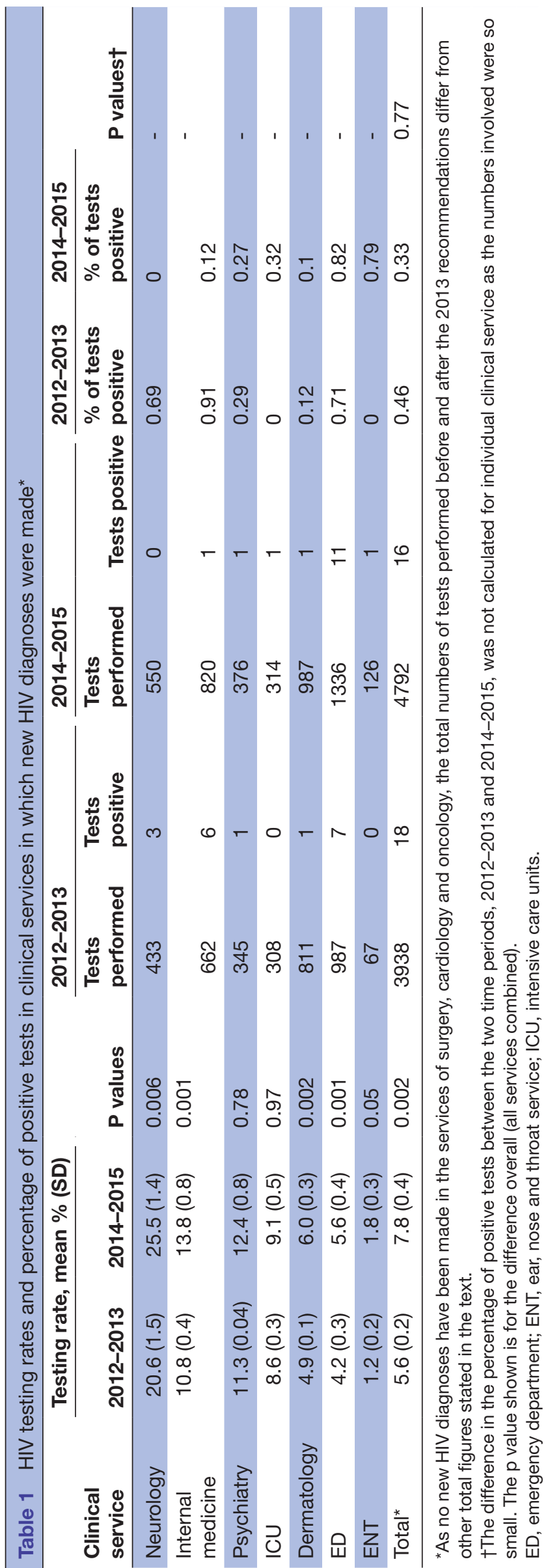


A

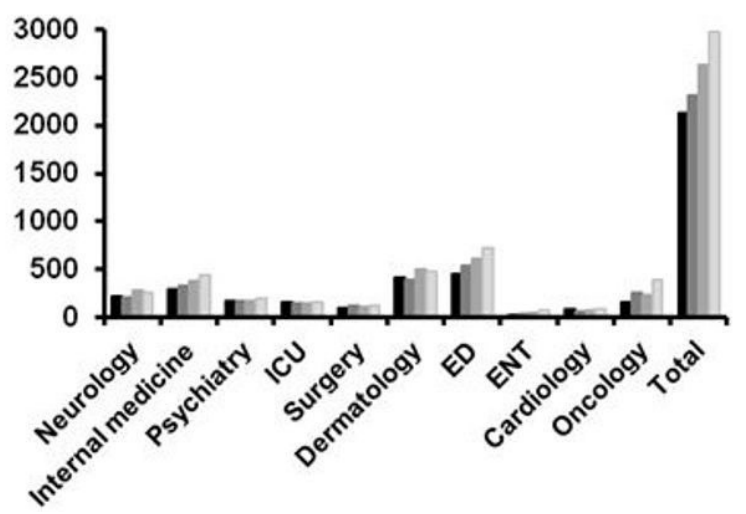

- 2012

$=2013$

॥ 2014

2015

\section{B}

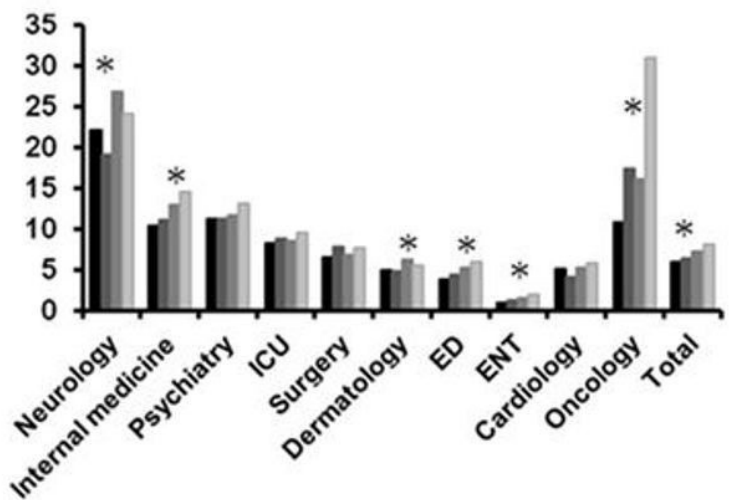

- 2012

a 2013

표 2014 $\square 2015$

Figure 1 Absolute number of HIV tests requested (panel A) and HIV testing rate (panel B) with time in the 10 clinical services studied. Asterisks indicate significant differences in rates $(p<0.01)$ between before $(2012-2013)$ and after (2014-2015) the publication of the Federal Office of Public Health HIV testing recommendations in November 2013. ED, emergency department; ENT, ear, nose and throat service; ICU, intensive care units.

(table 2). Testing rates were also significantly higher among male than among female patients in several clinical services and overall (table 2).

To examine patient origin, patients were pooled as Swiss and non-Swiss as the percentage of specific groups mentioned in the 2013 recommendations, notably patients from SSA, was low (online supplementary table 1 ). In several services, and overall, testing rates were significantly higher among non-Swiss compared with Swiss patients (table 2).

In the neurology service, testing practice changed with time. While there was a significant increase in testing rates between 2012 and 2015, the testing rates increased progressively among patients aged below 50 years while the testing rate among older patients remained stable (online supplementary figure 1). While progressive increases in testing rates were observed over time in other

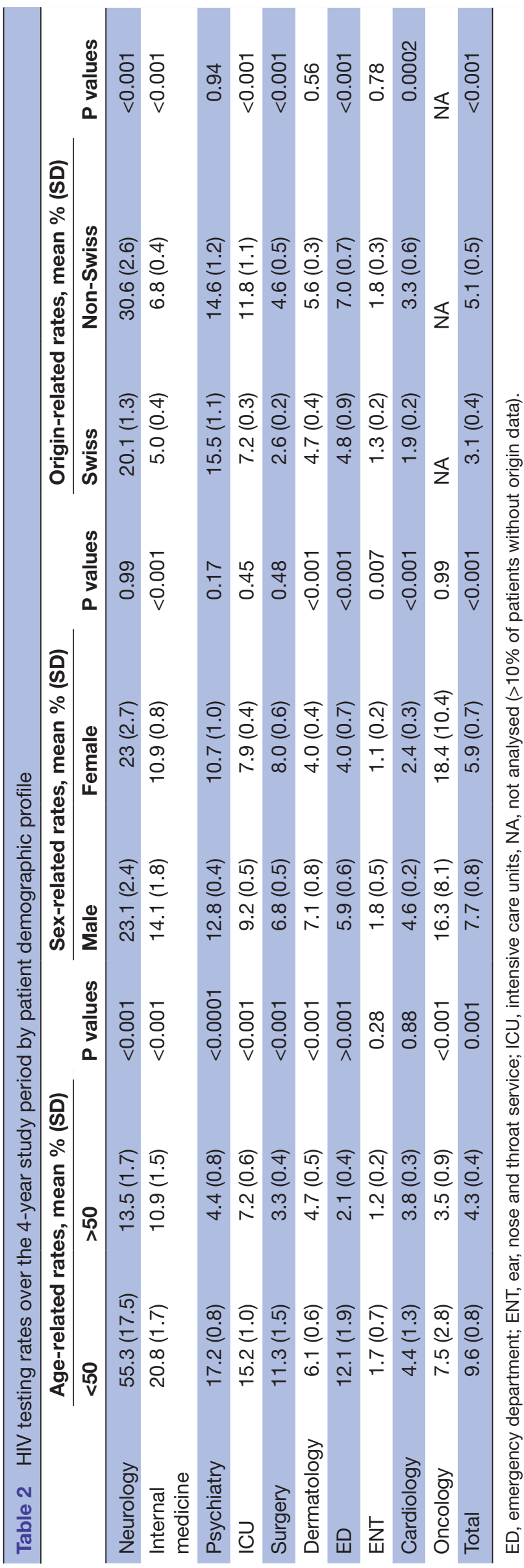




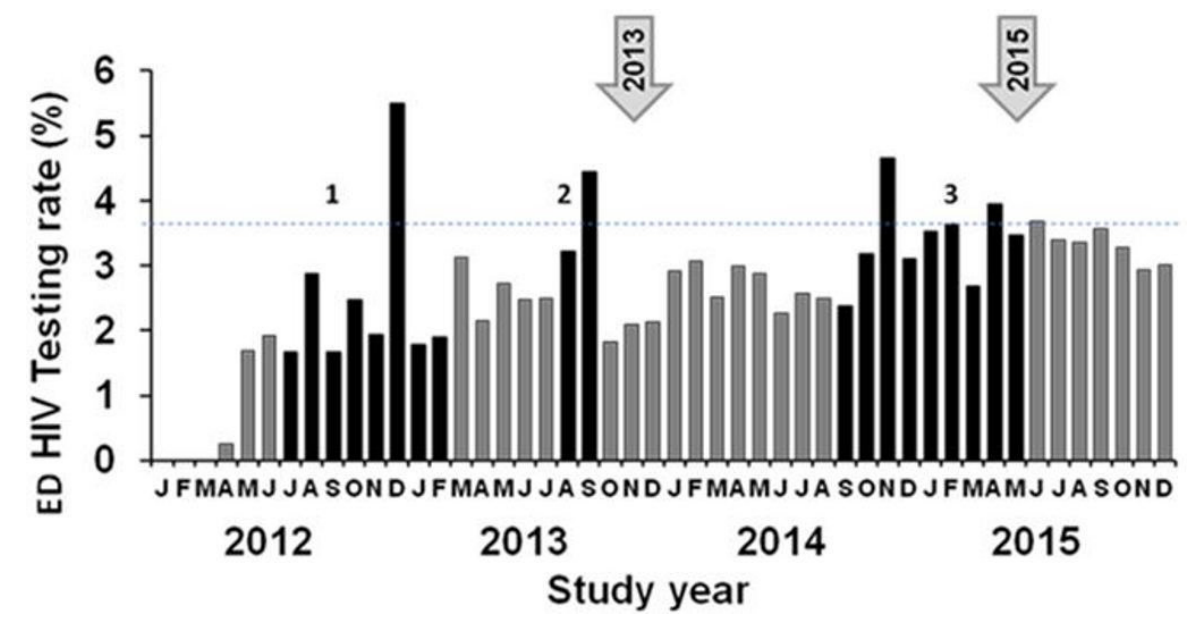

Figure 2 Monthly HIV testing rates in the emergency department (ED) sections involved in clinical trials on HIV testing and the temporal relationship between HIV testing rates and clinical trials (black bar groups 1 and 3), other training seminars (black bar group 2), and the publication of Federal Office of Public Health HIV testing recommendations (arrows 2013 and 2015 ). The dotted line indicates the mean testing rate for all ED sections for the year 2012, the first year of this study. ${ }^{1}$ Clinical trial examining patients' understanding of and attitudes to HIV testing in the ED. ${ }^{20}$ 2Training seminars on testing following the publication of low awareness of HIV testing recommendations among ED doctors in French-speaking Switzerland. ${ }^{14}{ }^{3}$ Clinical trial examining patient-associated and doctor-associated barriers to HIV testing in the ED and patient acceptance of rapid HIV testing. ${ }^{11}$

clinical services, no such increase was observed among patients with a specific demographic profile.

\section{Effect of local interventions}

During local educational interventions in ED sections involved in clinical trials, monthly testing rates exceeded the mean rate for the ED as a whole (figure 2). In the oncology service, testing rates increased significantly from $12 \%$ in 2012 to $16 \%$ in 2013 ( $\mathrm{p}=0.0005)$, when training seminars on HIV testing were provided to medical and nursing staff, in addition to increasing between before and after the 2013 recommendations as described above.

\section{DISCUSSION}

We have applied the same two-database tool with which we examined the effect of the $2010 \mathrm{FOPH}$ recommendations ${ }^{13}$ to examine the effect of the 2013 FOPH recommendations at our centre. We observed a global increase in HIV testing following the 2013 recommendations, with significant increases in clinical services receiving educational interventions in the context of clinical trials on testing. As these services had among the lowest testing rates prior to 2012, our observed increase suggests that local engagement complements the effect of national testing recommendations.

As we observed in our previous study, testing rates in the neurology, internal medicine and psychiatry IP services were higher than those in other services. ${ }^{13}$ These three services see patients presenting with many of the conditions listed as testing indications in the 2013 recommendations. Further, the neurology and internal medicine IP services make up two of the three principal services which receive patients admitted via the $\mathrm{ED}(\mathrm{OH}$, departmental data). Conversely, the cardiology service had relatively low testing rates. While some patients attending this service may have presented with non-vascular cardiac pathology or with vascular pathology presenting non-acutely, the low testing rates are surprising given accumulating evidence of a pathophysiological link between HIV infection and endothelial damage. ${ }^{21} 22$ However, while myocardial infarction, together with cerebrovascular events and peripheral arterial disease, has been mentioned as an indication for HIV testing in the 2010 FOPH recommendations, ${ }^{3}$ it is not mentioned in the 2013 update, ${ }^{4}$ and patients presenting with acute coronary syndromes to the LUH cardiology service are not tested routinely (OM, personal communication). Among cardiology patients who were tested over the 4-year period, the rate of new HIV diagnoses was zero. The finding that testing rates have been lower among dermatology patients than among surgery patients is also surprising. However, this may be explained by the high patient denominator in the dermatology service, and the possibility that patients seen have not presented HIV testing indications. All these observations highlight the importance of applying the two-database tool only when service structure (patient diagnosis profile, referral source, etc) is known, so that testing rates can be interpreted meaningfully.

In the ED, the testing rates were markedly higher than those reported previously. ${ }^{13}{ }^{14}$ In addition to the significant increase between before and after the recommendations, we observed a possible effect of local interventions in the form of training seminars and clinical trials. The positive effect of local interventions on HIV testing practice has been described in a London university hospital 
ED setting, the authors adding that constant commitment was required to achieve sustainability. ${ }^{23}$

We observed that testing was significantly higher among non-Swiss than Swiss patients in some services. Testing rates differed with other demographic characteristics, being higher among male patients and those aged below 50 years. Due to the retrospective design of this study, we could not identify why testing rates among Swiss, older and female patients were lower in some services, and it is possible that these patients did not present HIV testing indications. It is noteworthy, however, from Swiss HIV Cohort Study data, that older age and female sex were observed as risk factors for late presentation among patients newly diagnosed with HIV between 2009 and 2012. ${ }^{12}$ Perhaps future updates of Swiss HIV testing recommendations should mention the demographic profiles of individuals at risk of late presentation, to avoid undertesting patients not classically considered as high risk. ${ }^{1}$

This study has limitations. An important limitation was that the denominator used to calculate testing rates was taken as the number of patients presenting, with no means of identifying the number of patients who had HIV testing indications. We restricted our analysis to well-characterised subunits within each clinical service to optimise the percentage of patients presenting who had testing indications but this remains a limitation of our study. As with any single-centre study, the results presented may not be applicable to other settings, particularly if service structures differ. Although this two-database tool enables testing rates to be calculated and compared over time, it does not provide a 'margin for improvement' in each setting. We have no data on the percentage of healthcare professionals aware of the FOPH HIV testing recommendations, outside the ED setting, or the percentage of patients presenting testing indications who were not tested. In this way, while we observed improved testing rates with time, it is possible that this occurred through factors unrelated to the publication of the $2013 \mathrm{FOPH}$ HIV testing recommendations.

In conclusion, we have described a simple two-database method of measuring testing rates which enables healthcare centres to identify clinical services in which testing rates can be optimised and to follow progress with time. HIV testing rates increased at our centre over time, particularly in services involved in clinical trials on testing. The lower testing rates we observed among Swiss, older and female patients merit examination, given that patients lacking the 'classical' demography for HIV acquisition should still be included in testing initiatives if they present with indications.

\section{Author affiliations}

${ }^{1}$ Faculty of Biology and Medicine, University of Lausanne, Lausanne University Hospital, Lausanne, Switzerland

${ }^{2}$ Information and Management Control, Lausanne University Hospital, Lausanne, Switzerland

${ }^{3}$ Service of Immunology and Allergy, Lausanne University Hospital, Lausanne, Switzerland
${ }^{4}$ Service of Neurology, Lausanne University Hospital, Lausanne, Switzerland ${ }^{5}$ Service of Oncology, Lausanne University Hospital, Lausanne, Switzerland ${ }^{6}$ Cardiology Service, Lausanne University Hospital, Lausanne, Switzerland ${ }^{7}$ Emergency Department, Lausanne University Hospital, Lausanne, Switzerland ${ }^{8}$ Infectious Diseases Service, Lausanne University Hospital, Lausanne, Switzerland

Contributors TL contributed to study design, data collection and manuscript preparation. SM and RM contributed to data collection, data analysis and critical review. RADP, SP, MP and OM contributed to critical review. OH contributed to manuscript preparation and critical review. MC contributed to study design, manuscript preparation and critical review. KEAD contributed to study design, data collection, data analysis, manuscript preparation and critical review.

Funding This work was funded by the Infectious Diseases Service using existing funds and the Faculty of Biology and Medicine at the University of Lausanne.

Competing interests None declared.

Patient consent Not required.

Ethics approval Ethics Committee on Human Research of the Canton of Vaud, Switzerland (protocol number 2016-00368).

Provenance and peer review Not commissioned; externally peer reviewed.

Data sharing statement The database used for the analyses performed in the study is available on request.

Open access This is an open access article distributed in accordance with the Creative Commons Attribution Non Commercial (CC BY-NC 4.0) license, which permits others to distribute, remix, adapt, build upon this work non-commercially, and license their derivative works on different terms, provided the original work is properly cited, appropriate credit is given, any changes made indicated, and the use is non-commercial. See: http://creativecommons.org/licenses/by-nc/4.0/.

\section{REFERENCES}

1. Darling KE, Hachfeld A, Cavassini M, et al. Late presentation to HIV care despite good access to health services: current epidemiological trends and how to do better. Swiss Med Wkly 2016;146:w14348.

2. Office fédéral de la santé publique. Dépistage du VIH et conseil initiés par les médecins. Bulletin de l'OFSP 2007;21:371-3.

3. Office fédéral de la santé publique. Dépistage du VIH effectué sur l'initiative des médecins: recommandations pour les patients adultes. Bulletin de l'OFSP 2010:364-6.

4. Office fédéral de la santé publique. Dépistage du VIH effectué sur l'initiative des médecins en présence de certaines pathologies (maladies évocatrices d'une infection à VIH). Bulletin de I'OFSP 2013:1-5.

5. Office fédéral de la santé publique. Dépistage du VIH effectué sur l'initiative des médecins. Bulletin de /'OFSP 2015;21:237-41.

6. Branson BM, Handsfield HH, Lampe MA, et al. Revised recommendations for HIV testing of adults, adolescents, and pregnant women in health-care settings. MMWR Recomm Rep 2006;55:1-17.

7. Haute Authorité de Santé, 2009. Dépistage de l'infectino par le VIH en France https://www.has-sante.fr/portail/upload/docs/application/ pdf/2009-10/synthese_depistage_vih_volet_2_vfv_2009-10-21_1648-3_460.pdf.

8. British HIV Association, British Association of Sexual Health and HIV, British Infection Society, 2008. UK National guidelines for hiv testing http://www.bhiva.org/documents/Guidelines/Testing/ GlinesHIVTest08.pdf.

9. Burke RC, Sepkowitz KA, Bernstein KT, et al. Why don't physicians test for HIV? A review of the US literature. AIDS 2007;21:1617-24.

10. Hecht CR, Smith MD, Radonich K, et al. A comparison of patient and staff attitudes about emergency department-based HIV testing in 2 urban hospitals. Ann Emerg Med 2011;58:S28-S32.e4.

11. De Rossi N, Dattner N, Cavassini M, et al. Patient and doctor perspectives on HIV screening in the emergency department: A prospective cross-sectional study. PLoS One 2017;12:e0180389.

12. Hachfeld A, Ledergerber $\mathrm{B}$, Darling $\mathrm{K}$, et al. Reasons for late presentation to HIV care in Switzerland. J Int AIDS Soc 2015;18:20317.

13. Darling KE, Hugli O, Mamin R, et al. HIV testing practices by clinical service before and after revised testing guidelines in a Swiss University Hospital. PLoS One 2012;7:e39299.

14. Darling KE, de Allegri N, Fishman D, et al. Awareness of HIV testing guidelines is low among Swiss emergency doctors: a survey of 
five teaching hospitals in French-speaking Switzerland. PLoS One 2013;8:e72812.

15. Mosimann V, Cavassini M, Hugli O, et al. Patients with AIDS-defining cancers are not universally screened for HIV: a 10-year retrospective analysis of HIV-testing practices in a Swiss university hospital. HIV Med 2014;15:n/a.

16. Merz L, Zimmermann S, Peters S, et al. Investigating barriers in hiv-testing oncology patients: The ibitop study, phase I. Oncologist 2016;21:1176-82.

17. Sullivan AK, Raben D, Reekie J, et al. Feasibility and effectiveness of indicator condition-guided testing for HIV: results from HIDES I (HIV indicator diseases across Europe study). PLoS One 2013;8:e52845.

18. Kohler P, Schmidt AJ, Cavassini M, et al. Swiss HIV Cohort Study. The HIV care cascade in Switzerland: reaching the UNAIDS/WHO targets for patients diagnosed with HIV. AIDS 2015;29:2509-15.
19. UNAIDS. Epidemiology figures. Switzerland, 2013. http://www. unaids.org/sites/default/files/epidocuments/CHE.pdf2013.

20. Favre-Bulle T, Baudat D, Darling KE, et al. Patients' understanding of blood tests and attitudes to HIV screening in the emergency department of a Swiss teaching hospital: a cross-sectional observational study. Swiss Med Wkly 2015;145:w14206.

21. Freiberg MS, Chang CC, Kuller LH, et al. HIV infection and the risk of acute myocardial infarction. JAMA Intern Med 2013;173:614-22.

22. Nou E, Lo J, Grinspoon SK. Inflammation, immune activation, and cardiovascular disease in HIV. AIDS 2016;30:1495-509.

23. Rayment M, Rae C, Ghooloo F, et al. Routine HIV testing in the emergency department: tough lessons in sustainability. HIV Med 2013;14:6-9. 